\title{
EI Proceso Educativo Fiscal Y Su Influencia En Los Resultados De Las Pruebas Ser Estudiante, Del Séptimo Año De Educación General Básica, En El Cantón Guaranda, Ecuador
}

\author{
René Villacrés Borja \\ Mónica Bonilla Manobanda \\ Javier García López \\ Fidel Castro Bério
}

Universidad Estatal de Bolívar UEB, Facultad de Ciencias Administrativas Gestión Empresarial e Informática, Campus Académico "Alpachaca” Av.

Ernesto Che Guevara s/n y Av. Gabriel Secaira, Guaranda, Ecuador

Doi: 10.19044/esj.2017.v13n28p254 URL:http://dx.doi.org/10.19044/esj.2017.v13n28p254

\begin{abstract}
The Being Student tests recognize the characteristics of the students of the National Education System in relation to their knowledge. It allows the evaluated ones to know what situation they entail in the learning standards of each level, and was issued by the Ministry of Education. This therefore is aimed at knowing the level of learning achieved according to the Quality Standards issued by the Ministry of Education for this level. It is also in relation to their knowledge and reasoning skills. These instruments, according to their results, have allowed the Social Categorization of Educational Units at the National level, evidencing that the Educational Institutions of the Bolivar Province, especially the Guaranda Canton, are not within the first places of this social recognition. As a result, this implies that the fiscal education process up to this level is not meeting the standards of educational quality within the domain of knowledge. The objective of this study is to characterize how the fiscal education process influences the results of the student tests at the seventh year of basic general education. The unit of analysis is the educational process and the unit of observation is a student for each subject of each fiscal institution studied. The present research uses both quantitative and qualitative approach. This type of research is a documentary, bibliographical, and field research since it analyzed the notebooks of theoretical notes of duties, use of the official books for the academic period 2016 - 2017, and corrections of written tests
\end{abstract}


and their structure which serves as the key elements to determine and conceptualize the academic process.

Keywords: Student Testing, fiscal educational process, social categorization

\section{Resumen}

Las pruebas Ser Estudiante reconocen las características de las y los estudiantes del Sistema Nacional de Educación en relación con sus saberes y permite a los evaluados conocer en qué situación se encuentran con base en los estándares de aprendizaje de cada nivel, emitidos por el Ministerio de Educación, con el propósito de conocer el nivel de aprendizaje alcanzado de acuerdo a los Estándares de Calidad emitidos por el Ministerio de Educación para este nivel, en relación a sus saberes y habilidades de razonamiento. Dichos instrumentos según sus resultados han permitido la Categorización Social de las Unidades Educativas a nivel Nacional, evidenciándose que las Instituciones Educativas Fiscales de la Provincia Bolívar, en especial del Cantón Guaranda, no se encuentran dentro de los primeros lugares de este reconocimiento social; lo que implica que el proceso educativo fiscal hasta este nivel no está cumpliendo los estándares de calidad educativa dentro del dominio del conocimiento, el objetivo es caracterizar como el proceso educativo fiscal influye en los resultados de las pruebas Ser Estudiante, del séptimo año de educación general básica, la unidad de análisis es el proceso educativo y la unidad de observación es un estudiante por cada asignatura de cada institución fiscal estudiada. La presente investigación tiene el enfoque Cuantitativa y Cualitativa, el tipo de investigación es documental, bibliográfica y de campo ya que se analizó los cuadernos de apuntes teóricos, de deberes, utilización de los libros oficiales para el periodo académico 2016 - 2017, las correcciones de pruebas escritas y su estructura, que son elementos claves para determinar y conceptualizar al proceso académico.

Palabras-clave: Pruebas Ser Estudiante, proceso educativo fiscal, categorización social

\section{Introducción}

En nuestro país, desde 1996 hasta el año 2007, se han aplicado, en cuatro ocasiones, las pruebas APRENDO a los estudiantes de los años: tercero, séptimo y décimo de Educación Básica del sistema escolarizado, en las áreas de Matemática y Lenguaje y Comunicación. Estas pruebas se aplicaron de manera muestral y estuvieron fundamentadas en la Teoría Clásica de los Tests (Spearman, 1913; Gulliksen, 1950; Birnbaum, 1967; y Lord \& Novick, 1968). 
En este contexto, el Ministerio de Educación oficializó a partir del 4 de junio de 2008, la implementación de las pruebas SER Ecuador, para la evaluación del desempeño de los estudiantes, con la adopción de una nueva metodología: la Teoría de Respuesta al Ítem (Hambleton \& Swaminathan, 1985; Lord, 1980; Martínez-Arias, 1995; Muñiz, 1997) en el desarrollo de las pruebas de Logros Académicos y los cuestionarios de Factores Asociados. Por primera vez se aplicó estas pruebas en el año 2008, de manera censal, a estudiantes de establecimientos educativos fiscales, fiscomisionales, municipales y particulares, en los años: cuarto, séptimo y décimo de Educación Básica, y tercero de Bachillerato, en las áreas de Matemática y Lenguaje y Comunicación, y se incluyó las áreas de Estudios Sociales y Ciencias Naturales, de manera muestral, en los años: séptimo de Educación Básica.

La prueba Ser Estudiante - Séptimo EGB está dirigido a estudiantes que cursan este año de estudios siendo su objetivo determinar los niveles de logro en los estudiantes referidos a los Estándares de Calidad Educativa, siendo su impacto para el sujeto evaluado Bajo.

El tipo de prueba es Criterial de sensibilidad de instrucción Alta, con 85 ítems de opción múltiple y evalúa cuatro campos: Matemática, Lengua y Literatura, Ciencias Naturales y Estudios Sociales, la modalidad de aplicación es digital en 3 horas por campo durante un día y no se permite el uso de calculadora. (Ministerio de educación, 2011)

Este proceso de evaluación lleva adelante el Instituto Nacional de Evaluación Educativa (INEVAL) cuya misión es "Promover una educación de excelencia a través de la evaluación integral al Sistema Nacional de Educación y sus componentes" (Informate Ecuador, 2009), mediante la aplicación de evaluaciones confiables, objetivas, oportunas, pertinentes e imparciales y que toman como referencia los estándares de calidad educativa definidos por el Ministerio de Educación y enfocados en el aprendizaje de los estudiantes, amparados en la Constitución de la República en los artículos: 343, 344, 345 y especialmente en el artículo 346 que textualmente dice: "Existirá una institución pública, con autonomía de evaluación integral interna y externa, que promueva la calidad de la educación". (Asamblea Nacional, 2008)

De acuerdo con (Ministerio de educación, 2012) "Los estándares de calidad educativa son descripciones de los logros esperados correspondientes a los diferentes actores e instituciones del sistema educativo. En tal sentido, son orientaciones de carácter público que señalan las metas educativas para conseguir una educación de calidad", en nuestro caso se ha analizado el estándar de aprendizaje que son descripciones de los logros obtenidos por los estudiantes y constituyen referentes comunes que deben alcanzar a lo largo 
de su trayectoria escolar; desde el primer grado de la Educación General Básica hasta el tercer curso de Bachillerato.

Los estándares evaluados corresponden a las cuatro áreas básicas que son: Lengua y Literatura, Matemática, Estudios Sociales y Ciencias Naturales, en este estudio corresponde al Tercer nivel de progresión, dentro de los estándares de cada área, se proponen dominios del conocimiento, los cuales expresan los núcleos de aprendizaje y destrezas centrales del área curricular que desarrollan procesos de pensamiento, a partir de la comprensión y aplicación de los conocimientos esenciales.

Mientras tanto El currículo Nacional contiene las herramientas necesarias para que el estudiante de cada año lectivo, pueda ir aproximándose a estos estándares, en consecuencia, si se aplica el Currículo Nacional de manera adecuada, los estudiantes alcanzarán los estándares de aprendizaje.

El Ministerio de Educación en el sistema nacional de evaluación al Desempeño del estudiante, "pretende medir las actitudes y aptitudes del estudiante como respuesta al proceso educativo; es decir, las demostraciones de los conocimientos, habilidades, destrezas y valores desarrollados, como resultado del proceso educativo y su aplicación a la vida cotidiana". (Ministerio, 2012)

Según el (Ministerio, 2012) "Establecen la manera cómo un plantel educativo se organiza y desarrolla procesos de gestión para mejorar la calidad de los aprendizaje de los estudiantes". Por otro lado estos estándares ayudan a que los docentes, autoridades, estudiantes y padres de familia se desarrollen y contribuyan al óptimo funcionamiento de las instituciones educativas.

Añade además que "se espera que los agentes educativos sepan: analizar las situaciones para la toma de decisiones, comunicar efectivamente a todos los miembros de la comunidad, manejar los conflictos, liderar y orientar a la comunidad educativa", para trabajar conjuntamente de forma reflexiva, práctica llegando a acuerdos consensuados.

Para ello las instituciones educativas, teniendo conocimiento de su realidad, serán capaces de establecer acciones y planes de mejora necesarios.

Dentro de la gestión del aprendizaje se considera los siguientes estándares generales que el docente debe realizar dentro del aula:

- $\quad$ Planifica para el proceso de enseñanza-aprendizaje.

- Implementa procesos de enseñanza-aprendizaje en un clima que promueve la participación y el debate.

- $\quad$ Evalúa, retroalimenta e informa acerca de los procesos de aprendizaje de sus estudiantes. (Ministerio, 2012)

Para complementar este proceso se toma en cuenta el ambiente del aula donde el docente genere un adecuado clima de aprendizaje a través de 
instrucciones claras y de la aplicación de métodos para la enseñanzaaprendizaje fortaleciendo con su reflexión y práctica cotidiana.

Según (Pérez, 2017) realiza una clasificación de los mejores colegios a nivel nacional de acuerdo al puntaje obtenido por los estudiantes en la prueba Ser Bachiller del periodo 2015-2016, en la que sobresalen las instituciones privadas y mixtas; lo que llama la atención es que las Instituciones Educativas Fiscales y las Unidades del Milenio no se encuentran en esta categorización.

Dentro de esta categorización social se encuentra la Unidad Educativa Verbo Divino, de la provincia Bolívar, la que se ubica en el segundo lugar a nivel nacional.

Ante esta realidad de nuestras instituciones educativas fiscales se plantea el siguiente problema: De qué manera el proceso educativo fiscal influye en los resultados de las pruebas Ser Estudiante séptimo año de EGB en el cantón Guaranda.

Cuyo Objetivo es: Caracterizar como el proceso educativo fiscal influye en los resultados de las pruebas Ser Estudiante, del séptimo año de educación general básica, en el cantón Guaranda, analizando el proceso educativo con las siguientes variables:

Tabla $\mathrm{N}^{\circ} 1$. Variables analizadas

\begin{tabular}{|c|c|c|c|c|}
\hline $\begin{array}{c}\text { VARIABLE } \\
\text { INDEPENDIENTE }\end{array}$ & $\begin{array}{l}\text { Variables } \\
\text { Intermedias }\end{array}$ & \multicolumn{2}{|c|}{ Variables Respuesta } & Instrumento \\
\hline \multirow{4}{*}{ Proceso Educativo } & $\begin{array}{l}\text { Instrumentos de } \\
\text { Evaluación }\end{array}$ & Tipos de prueba & $\begin{array}{c}\text { Objetivas } \\
\text { No Objetivas }\end{array}$ & $\begin{array}{c}\text { Guía de } \\
\text { observación. }\end{array}$ \\
\hline & Conocimientos & Contenidos & $\begin{array}{l}\text { Nacional } \\
\text { INEVAL }\end{array}$ & $\begin{array}{c}\text { Guía de } \\
\text { observación. }\end{array}$ \\
\hline & Destrezas & Cumplimiento & Asignatura & $\begin{array}{c}\text { Guía de } \\
\text { observación. }\end{array}$ \\
\hline & Aplicación & $\begin{array}{l}\text { Teórico } \\
\text { Practico }\end{array}$ & $\begin{array}{c}\text { Por cada } \\
\text { asignatura }\end{array}$ & $\begin{array}{c}\text { Guía de } \\
\text { observación. }\end{array}$ \\
\hline
\end{tabular}

Elaborado por: Los autores

Consideramos la siguiente Hipótesis: El proceso educativo fiscal evidenciado a través del cuaderno de materia influye favorablemente en los resultados de las pruebas Ser Estudiante, del séptimo año de educación general básica, en el cantón Guaranda.

Se considera al salón de clase como medio fundamental donde existe la relación directa psicopedagógica entre el docente - estudiante y es aquí que se despliega sus recursos personales y didácticos para cumplir con su labor académica, por lo tanto el aprendizaje en el aula es entendido como una práctica pedagógica deliberada, sistemática, modelada e intensiva dirigida a un grupo de estudiantes que se encuentran en situación de desarrollo y aprendizaje.

Estableciéndose que uno de los productos de esta relación es el cuaderno de materia y que están concebidos como verdaderos instrumentos 
de trabajo de los estudiantes en los cuales éstos construyen y ponen en juego sus capacidades de comprender, relacionar, transferir un aprendizaje a otro contexto, sintetizar, buscar información y, por lo tanto, en ellos escriben, subrayan, dibujan, grafican, registran experiencias, que refleja el trabajo en clase, siendo evidencia para la evaluación continua; a través de él se puede comprobar las siguientes actividades registradas por el estudiante:

- Toma apuntes correctamente.

- $\quad$ Su nivel de comprensión, de abstracción y que ideas selecciona.

- $\quad$ Su nivel de expresión escrita, la claridad y propiedad de sus expresiones.

- La ortografía, caligrafía, comprensión, composición de frases.

- $\quad$ Si realiza esquemas, resúmenes, subrayados.

- $\quad$ El cuidado o dedicación que emplean en llevar al día su cuaderno.

\section{Además}

- $\quad$ El cuaderno sirve al estudiante como material de apoyo en su preparación para las pruebas escritas de evaluación mediante la revisión de las actividades realizadas por él.

- $\quad$ Que el alumno conozca los aspectos de su proceso de aprendizaje y le permita mejorar a través de la heteroevaluación.

- Que el alumno tenga un material con contenidos que sirvan para trabajar en otras asignaturas.

\section{Material y Métodos}

El estudio tiene el enfoque Cuanti-Cualitativo y se apoya metodológicamente con el procedimiento de la investigación documental, bibliográfica y de campo ya que se analizó los cuadernos de apuntes teóricos, de deberes, utilización de los libros oficiales para el periodo académico 2016 - 2017, las correcciones de pruebas escritas y su estructura, que son elementos claves para determinar y conceptualizar al proceso académico y que constituye la unidad de análisis; por otra parte la unidad de observación corresponde un estudiante por asignatura con los materiales educativos mencionados, en las asignaturas siguientes: Matemática; Lengua y Literatura; Ciencias Sociales y Ciencias Naturales del séptimo año de la Educación General Básica de las Unidades Educativas fiscales del Cantón Guaranda; mismas que fueron analizados con una ficha de observación científica no participante y estructurada de escala nominal y medida su grado de confiablidad a través Kuder-Richardson (KR-20) y cuyo resultado es de 0,82 computado en hoja de Excel 2016 y 0,92 el Alfa de Cronbach calculado en SPSS 22. 


\section{Resultados y Discusión}

Se puede evidenciar que en los cuadernos de trabajo o materia no existe la corrección de las pruebas aplicadas a los estudiantes, en un $75 \%$, comprobándose que la heteroevaluación no se aplica dentro del proceso educativo.

En la estructura de las pruebas en un $50 \%$ predomina preguntas conceptuales de las asignaturas, en un 44\% la estructura es de opción múltiple, sin embargo no se toma en cuenta la estructura de las pruebas preparadas por el INEVAL.

Los apuntes teóricos en las asignaturas en un 50\% predominan los conceptos teóricos, seguido en un $37 \%$ con ejercicios simples y en un $13 \%$ problemas aplicados.

En relación a la apariencia de los cuadernos y libros en un $81 \%$ de muy bueno y en el $19 \%$ manifiesta que es regular.

En los cuadernos de apuntes teóricos en todas las asignaturas en un $63 \%$ no existe la corrección de las faltas ortográficas por parte del profesor, limitándose a una revisión superficial de cumplimiento.

Los mapas conceptuales son una herramienta importante de didáctica en el aula, sin embargo se pudo notar que éstos no existen en un 56\%, lo que produce un aprendizaje en forma desorganizada sin jerarquización.

En los cuadernos se pudo observar que existe una comunicación gráfica en relación con la teoría y la aplicación en un $88 \%$.

Una de las formas de revisión del cumplimiento de las tareas es la rúbrica del docente en el cuaderno del estudiante, esto se pudo visualizar en un $75 \%$ de estos.

En referencia a la calificación de las tareas en el cuaderno de los estudiantes, un $88 \%$ posee la misma y un $12 \%$ no lo tiene.

Los libros oficiales entregados por el gobierno en un $94 \%$ son utilizados por los maestros y el $6 \%$ no los utiliza, contraviniendo las disposiciones oficiales.

\section{Comprobación de Hipótesis}

$\mathrm{H}_{\mathrm{o}}$ : El proceso educativo fiscal evidenciado a través del cuaderno de materia no influye favorablemente en los resultados de las pruebas Ser Estudiante, del séptimo año de educación general básica, en el cantón Guaranda.

$\mathrm{H}_{\mathrm{a}}$ : El proceso educativo fiscal evidenciado a través del cuaderno de materia si influye favorablemente en los resultados de las pruebas Ser Estudiante, del séptimo año de educación general básica, en el cantón Guaranda. 
Para la prueba de la hipótesis se ha utilizado la Prueba no paramétrica $\mathrm{Q}$ de Cochran para muestras relacionadas de nivel nominal y utilizando el programa estadístico Spss 22, sus resultados son:

Tabla $\mathrm{N}^{\circ} 2$. Resultados de la prueba de hipótesis

\begin{tabular}{|c|c|c|c|c|}
\hline Tipo & Calor & V4 & V5 \\
\hline 0 & Corrección pruebas & 25 & $025 \% 175 \%$ & Corrección pruebas \\
\hline 0 & Tipo prueba & 75 & $075 \% 125 \%$ & Tipo prueba \\
\hline 0 & Relación & 56 & $056 \% 144 \%$ & Relación \\
\hline 0 & Apariencia & 81 & $081 \% 119 \%$ & Apariencia \\
\hline 0 & Corrección ortografía & 37 & $037 \% 163 \%$ & Corrección ortografía \\
\hline 0 & Mapas conceptuales & 43 & $043 \% 157 \%$ & Mapas conceptuales \\
\hline 0 & Comunicación gráfica & 87 & $087 \% 113 \%$ & Comunicación gráfica \\
\hline 0 & Rubrica profesor & 87 & $087 \% 113 \%$ & Rubrica profesor \\
\hline 0 & Calificación & 87 & $087 \% 113 \%$ & Calificación \\
\hline 0 & Utiliza libro & 81 & $081 \% 119 \%$ & Utiliza libro \\
\hline 1 & Corrección pruebas & 75 & $025 \% 175 \%$ & Corrección pruebas \\
\hline 1 & Tipo prueba & 25 & $075 \% 125 \%$ & Tipo prueba \\
\hline 1 & Relación & 44 & $056 \% 144 \%$ & Relación \\
\hline 1 & Apariencia & 19 & $081 \% 119 \%$ & Apariencia \\
\hline 1 & Corrección ortografía & 63 & $037 \% 163 \%$ & Corrección ortografía \\
\hline 1 & Mapas conceptuales & 57 & $043 \% 157 \%$ & Mapas conceptuales \\
\hline 1 & Comunicación gráfica & 13 & $087 \% 113 \%$ & Comunicación gráfica \\
\hline 1 & Rubrica profesor & 13 & $087 \% 113 \%$ & Rubrica profesor \\
\hline 1 & Calificación & 13 & $087 \% 113 \%$ & Calificación \\
\hline 1 & Utiliza libro & 19 & $081 \% 119 \%$ & Utiliza libro \\
\hline & & & 19 & \\
\hline
\end{tabular}

Fuente: Trabajo de campo

Elaborado por: Los autores

\section{Gráfico $N^{\circ}$ 1: Prueba $Q$ de Cochran para muestras relacionadas}

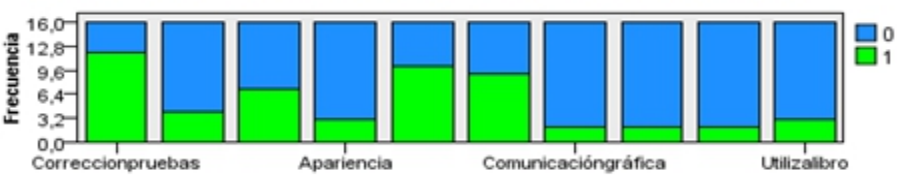

\begin{tabular}{|lr|}
\hline N total & 16 \\
\hline Estadistico de contraste & 59,567 \\
\hline Grados de libertad & 9 \\
\hline $\begin{array}{l}\text { Significación asintótica (prueba } \\
\text { bilateral) }\end{array}$ &, 000 \\
\hline
\end{tabular}

Tabla $\mathrm{N}^{\circ}$ 3. Resumen de Hipótesis

\begin{tabular}{|c|c|c|c|c|}
\hline & Hipótesis nula & Prueba & Sig. & Decisión \\
\hline 1 & $\begin{array}{l}\text { Las distribuciones de Corrección pruebas, Tipo } \\
\text { prueba, Relación, Apariencia, Corrección } \\
\text { ortografía, Mapas conceptuales, Comunicación } \\
\text { gráfica, Rubrica profesor, Calificación and } \\
\text { Utiliza libro son las mismas. }\end{array}$ & $\begin{array}{l}\text { Prueba Q de } \\
\text { Cochran para } \\
\text { muestras } \\
\text { relacionadas }\end{array}$ & $\mathbf{0 , 0 0 0}$ & Rechace la hipótesis nula. \\
\hline
\end{tabular}

Se muestran significaciones asintóticas. El nivel de significancia es 0,05 


\section{Decisión}

Al ser el valor significativo $0,000 \leq 0,05$, entonces la hipótesis nula se rechaza y se acepta la alterna, es decir: El proceso educativo fiscal evidenciado a través del cuaderno de materia si influye favorablemente en los resultados de las pruebas Ser Estudiante, del séptimo año de educación general básica, en el cantón Guaranda, con un nivel de confianza del 95\%.

El proceso educativo fiscal influye favorablemente en los resultados de las pruebas Ser Estudiante, del séptimo año de educación general básica, en el cantón Guaranda.

Con los resultados anteriormente descritos del proceso académico se ratifica la posición del décimo sexto lugar de nuestra provincia según informe del Instituto Nacional de Evaluación Educativa, Resultados educativos, retos hacia la excelencia, 2015-2016.

Gráfico $\mathrm{N}^{\circ} 2$ : Niveles de logro de $7^{\mathrm{mo}}$ grado por provincia

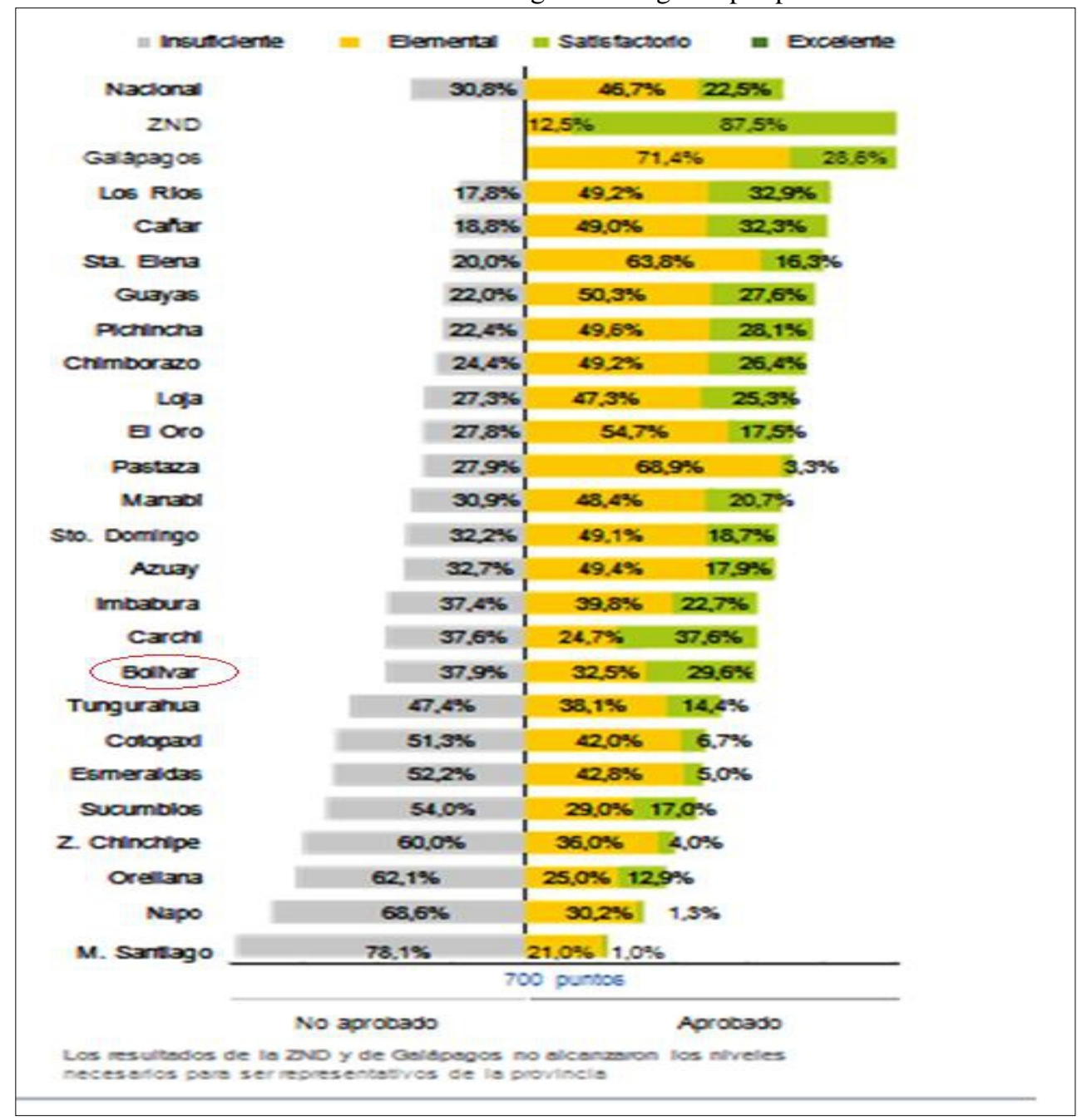




\section{Conclusion}

1. El docente incumple la normativa pedagógica en la aplicación del currículo nacional y los estándares de calidad exigidos para los estándares de aprendizaje.

2. En el proceso de enseñanza aprendizaje el docente no utiliza de forma eficiente los libros que entrega el gobierno.

3. Se deja de lado la utilización de la guía de la prueba Ser Estudiante para la aplicación de las pruebas dentro del aula y preparar al estudiante para su prueba final.

4. En los cuadernos de materia no se corrige las pruebas que se aplica para conocimiento del estudiante y procurar su mejor aprovechamiento.

5. Se deja de observar la importancia que tiene para el estudiante que en los cuadernos, el docente corrija las faltas cometidas y así motivar a su aprendizaje.

\section{References:}

1. Asamblea Nacional (2008). Constitución de la República del Ecuador. Quito: Registro Oficial.

2. Informate Ecuador (2009). http://informateecuador.com. Recuperado el 16 de julio de 2017, de http://informateecuador.com: http://informateecuador.com/resultados-ser-bachiller/

3. Ministerio de educación (2011). Actualización y fortalecimiento curricular de la educación general básica $7 m o$ año. Quito: Ministerio de educación.

4. Ministerio de educación (2012). estandádres de calidad educativa. Quito: Ministerio de educación.

5. Ministerio de educación (2012). ESTÁNDARES DE CALIDAD EDUCATIVA. Quito: MInisterio de educación.

6. Ministerio, E. (2012). educacion.gob.ec. Recuperado el 15 de julio de 2017, de educacion.gob.ec: https://educacion.gob.ec/desempeno-delestudiante/

7. Pérez, A. (2017). Los 100 Mejores Colegios Del País: ¿Dónde Esta La Calidad? Vistazo, 86-87. 